\title{
Fluid flow and pressure effects in phase-field models for solidification
}

\author{
M. Conti \\ Dipartimento di Fisica, Universita' di Camerino, Camerino, Italy
}

\begin{abstract}
We present an extension of the classic phase-field model to incorporate hydrodynamic effects and compressible fluid flow. The methods of the extended irreversible thermodynamics are used to derive the dynamic equations for the solidification process. We present also some numerical solutions of the model, which show to what extent the crystal growth deviates from the pure diffusive description of the process.
\end{abstract}

\section{Introduction}

The growth of a crystal from the melt is a complex phenomenon which involves many physical effects. The rejection of the latent heat (and solute, for alloy solidification), away from the solid-liquid interface is accompanied by the formation of thermal and solute boundary layers which strongly affect the morphological instability of the interface. Moreover across the interface the density changes, (the solid is generally denser than the liquid); the change ranges from a few percent for simple metals to more than $20 \%$ for some eutectic mixtures. The shrinking, or in some cases the dilatation, of the system causes an advection flow in the liquid phase. Then, even in absence of gravity, the purely diffusive picture for the conserved fields in the bulk phases should be extended, to incorporate hydrodynamic effects.

Flow effects in solidification may be treated through an extension of the phase-field model [1]. In the classic formulation of the model, a non-conserved order parameter $\phi(\mathbf{x}, t)$ characterizes the phase of the system at each point. A suitable free energy (or entropy) functional is then constructed, that depends on the order parameter as well as on the associate fields and their gradients. 
The dynamic equations for the process are derived through the extremization of the thermodynamic potential in respect to these variables.

In the present paper we present a model which incorporates flow effects due to density change. The solid phase is modeled as an isotropic fluid with large viscosity. The density is treated as an independent dynamic variable, related to the local pressure via an equation of state. The entropy production equation, coupled with the balance of mass, momentum and energy, is used to derive the governing equations for the relaxation of the system.

We present some numerical solutions of the model, which show the effects of the density changes and the liquid flow in crystal solidification. We address also the growth of a crystal from its supercooled liquid in a closed domain (constrained growth), assuming that the liquid expands upon solidification. In this case the growth is contrasted by an increasing pressure, which results in a continuous decrease of the effective supercooling; we observe that a quasi-static approximation is a satisfactory approach to interpret the growth dynamics.

\section{A phase-field model with hydrodynamics}

\subsection{The equations of the model}

The equations of the model are derived in detailed form in [2]; here we give a short review of the thermodynamic methods. The local state of a pure substance which undergoes solidification is characterised by a coarse grained density $\rho(\vec{x}, t)$, the local temperature $T$ and an order parameter $\phi(\vec{x}, t)$, which is assumed to take the values $\phi=0$ in the solid and $\phi=1$ in the liquid. The velocity field is denoted by $\vec{v}$, the specific energy by $e^{\prime}$ and the specific entropy by $s^{\prime}$. The two latter quantities are related to the specific free energy $f^{\prime}(\rho, \phi, T, \nabla \rho, \nabla \phi)$ through standard thermodynamic relations, and involve gradient contributions. The stress tensor is denoted by $\mathbf{P} ; \vec{J}_{\mathrm{E}}, \vec{J}_{\mathrm{S}}$ represent the energy and entropy flux vectors respectively, and $\sigma$ is the entropy production rate. Finally, $\vec{g}$ stands for a specific body force field. In terms of these variables the classical balance laws read:

$$
\begin{array}{r}
\frac{d \rho}{d t}=-\rho \nabla \cdot \vec{v} ; \quad \rho \frac{d \vec{v}}{d t}=\rho \vec{g}-\nabla \cdot \mathbf{P} ; \\
\rho \frac{d e^{\prime}}{d t}=-\nabla \cdot \vec{J}_{\mathrm{E}}-\mathbf{P}: \nabla \vec{v} ; \quad \rho \frac{d s^{\prime}}{d t}=-\nabla \cdot \vec{J}_{\mathrm{S}}+\sigma
\end{array}
$$

A generalised specific Helmoltz free energy is postulated as

$$
f^{\prime}(\rho, \phi, T, \nabla \rho, \nabla \phi)=f(\rho, \phi, T)+\frac{1}{2 \rho}\left[\delta_{\mathrm{F}}^{2}(\nabla \rho)^{2}+\epsilon_{\mathrm{F}}^{2}(\nabla \phi)^{2}\right]
$$

where $f(\rho, \phi, T)$ is the specific bulk free energy, and the the gradient terms account for the energy cost of the interfacial region. The differential form of the second law 
of thermodynamics, along with the balance equations (1) leads to a local form of the entropy production rate written as:

$$
\begin{gathered}
\sigma=-\frac{1}{T} \frac{d \phi}{d t}\left(\rho \frac{\partial f}{\partial \phi}-\epsilon_{\mathrm{F}}^{2} \nabla^{2} \phi\right)+\vec{J}_{\mathrm{E}} \cdot \nabla\left(\frac{1}{T}\right)+\frac{d \rho}{d t} \nabla \rho \cdot \nabla\left(\frac{\delta_{\mathrm{F}}^{2}}{T}\right) \\
+\frac{d \phi}{d t} \nabla \phi \cdot \nabla\left(\frac{\epsilon_{\mathrm{F}}^{2}}{T}\right)-\frac{1}{T}(\mathbf{P}+\mathbf{T}): \nabla \vec{v}
\end{gathered}
$$

where $\mathbf{T}$ is a tensor with components:

$$
T_{i k}=\delta_{i k}\left[-p+\rho \delta_{\mathrm{F}}^{2} \nabla^{2} \rho+\frac{1}{2} \delta_{\mathrm{F}}^{2}(\nabla \rho)^{2}+\frac{1}{2} \epsilon_{\mathrm{F}}^{2}(\nabla \phi)^{2}\right]-\delta_{\mathrm{F}}^{2} \rho_{i} \rho_{k}-\epsilon_{\mathrm{F}}^{2} \phi_{i} \phi_{k}
$$

where $\rho_{i}, \rho_{k}, \phi_{i}, \phi_{k}$ stay for $\partial \rho / \partial x_{i}, \partial \rho / \partial x_{k}, \partial \phi / \partial x_{i}, \partial \phi / \partial x_{k}$. Notice that this form of the tensor reduces to the classic capillary stress tensor at the solid-liquid interface.

Neglecting the thermal gradient across the interface, the third and fourth terms in Eq. (3) vanish, and the constraint of local positive entropy production requires:

$$
\frac{d \phi}{d t}=-\Gamma\left(\rho \frac{\partial f}{\partial \phi}-\epsilon_{\mathrm{F}}^{2} \nabla^{2} \phi\right)
$$

where $\Gamma$ is a positive constant. We recover also the standard thermal conduction law $\vec{J}_{\mathrm{E}}=-K \nabla T$, where $K$ is the thermal conductivity. Moreover, it is reasonable to assume that the entropy source in the last term of Eq. (3) is only due to viscous dissipation, then we obtain $\mathbf{P}=-\mathbf{T}-\boldsymbol{\Pi}$, with $\boldsymbol{\Pi}$ indicating the standard stress tensor for viscous fluids. To find an explicit expression for the specific free energy $f$, we assume that at equilibrium the latter should take the form of a double well over the $\rho, \phi$ plane, with two minima centered at the bulk solid $\left(\rho=\rho_{\mathrm{s} 0}, \phi=0\right)$ and liquid ( $\rho=\rho_{\mathrm{l} 0}, \phi=1$ ), where we denoted as $\rho_{\mathrm{s} 0}, \rho_{\mathrm{l} 0}$ the equilibrium densities in the two phases at the coexistence temperature $T_{0}$ and pressure $p_{0}$.

Moreover, the free energy must be consistent with the equation of state

$$
\rho-\rho_{0}=-\beta \rho_{0}\left(T-T_{0}\right)+k \rho_{0}\left(p+\tilde{P}-p_{0}\right)
$$

where $\beta$ is the thermal expansion coefficient and $k$ the isothermal compressibility; $p$ is the thermodynamic pressure given by $p=\rho^{2}(\partial f / \partial \rho)$ and $\tilde{P}$ is the excess pressure due to the capillary stress, written as $\tilde{P}=\epsilon_{\mathrm{F}}^{2}(\nabla \phi)^{2} / 2+\delta_{\mathrm{F}}^{2}(\nabla \rho)^{2} / 2-$ $\rho \delta_{\mathrm{F}}^{2} \nabla^{2} \rho$

A suitable expression of the free energy may be written as

$$
\begin{array}{r}
f(\rho, \phi, T)=\frac{a g(\phi)}{\rho}+\frac{\beta}{k}\left(T-T_{0}\right)\left[\frac{\rho-\rho_{0}(\phi)}{\rho \rho_{0}(\phi)}\right]+\left(p_{0}-\tilde{P}\right)\left[\frac{\rho-\rho_{0}(\phi)}{\rho \rho_{0}(\phi)}\right] \\
-C T \ln \frac{T}{T_{0}}+C\left(T-T_{0}\right)+p(\phi) L_{0}\left(1-\frac{T}{T_{0}}\right)-\frac{\left(p_{0}-\tilde{P}\right)}{\rho_{0}(\phi)}+\frac{\rho_{0}(\phi)}{2 k}\left[\frac{\rho-\rho_{0}(\phi)}{\rho \rho_{0}(\phi)}\right]^{2}
\end{array}
$$

with $g(\phi)=(1 / 4) \phi^{2}(1-\phi)^{2}$. In Equation (7) $C$ is the specific heat and $L_{0}$ the latent heat per unit mass in the reference state. We observe a Landau-Ginzburg 
contribution for the order parameter $\phi$ (first term in the r.h.s of the equation), and the elastic contribution of the last term. The function $p(\phi)$, monotonic and increasing with $\phi$, assumes the values $p(0)=0, p(1)=1$, and describes the transition of the free energy from the solid to the liquid phase. The equilibrium density $\rho_{0}(\phi)$ is assumed to change in the interfacial region as $\rho_{0} \equiv \rho_{0}(\phi)=$ $\rho_{\mathrm{s} 0}+p(\phi)\left(\rho_{\mathrm{l} 0}-\rho_{\mathrm{s} 0}\right)$. In the sequel we assume that all the bulk properties of the system change in the same fashion along the solid-liquid interface. Then, Equation (5) and the mass, momentum and energy balance represent the evolution equations for the system.

\subsection{The non-dimensional form}

A nondimensional form of the model equations is obtained adopting a reference length $\xi$ and scaling time to $\tau=\xi^{2} / D$, with $D$ indicating the thermal diffusivity. Density is scaled as $\rho / \rho_{10}$ and a nondimensional temperature is introduced as $u=C\left(T-T_{0}\right) / L_{0}$. Specific energies will be scaled to $v_{0}^{2}$, where $v_{0}=\xi / \tau$ is the natural reference for velocities, and the specific body force is scaled to $\xi^{2} / \tau$. The scale for the components of the stress tensor is $\rho_{10} v_{0}^{2}$. The equilibrium density takes the form $\rho_{0}=S+p(\phi)(1-S)$, with $S=\rho_{\mathrm{s} 0} / \rho_{\mathrm{l} 0}$. Retaining for simplicity the same symbols for the scaled (nondimensional) quantities, the model equations in two dimensions read:

$$
\begin{gathered}
\frac{d \rho}{d t}=-\rho \nabla \cdot \vec{v} \\
\rho \frac{d \vec{v}}{d t}=\rho \vec{g}+\nabla \cdot(\mathbf{T}+\mathbf{\Pi}) \\
\frac{d u}{d t}+p^{\prime}(\phi) \frac{d \phi}{d t}=\nabla^{2} u+\frac{1}{\rho R_{5}}(\mathbf{\Pi}: \nabla \vec{v})+R_{8} \frac{\tilde{\epsilon}}{\alpha} \frac{R_{1}}{m} \frac{1}{\rho_{0}^{2}} \frac{d \rho}{d t} \\
\frac{d \phi}{d t}=m\left\{\nabla \cdot\left(\eta^{2}(\theta) \nabla \phi\right)+\frac{\partial}{\partial y}\left[\eta(\theta) \eta^{\prime}(\theta) \phi_{x}\right]-\frac{\partial}{\partial x}\left[\eta(\theta) \eta^{\prime}(\theta) \phi_{y}\right]\right\} \\
-\frac{m}{\tilde{\epsilon^{2}}}\left[\frac{\partial g(\phi)}{\partial \phi}-p^{\prime}(\phi) \rho \alpha \tilde{\epsilon} u\right]-\frac{1}{2 \rho_{0}^{2}} p^{\prime}(\phi) \rho(1-S) R_{1}\left(\frac{\rho_{0}^{2}-\rho^{2}}{\rho^{2}}-2 R_{8} u\right)
\end{gathered}
$$

where the components of the capillary stress tensor are:

$$
\begin{aligned}
& T_{i k}=\delta_{i k}\left[-p_{0}-\frac{R_{1} R_{3}}{m}\left(R_{8} u+\frac{\rho-\rho_{0}}{\rho}\right)+R_{2}(\nabla \rho)^{2}+R_{3}(\nabla \phi)^{2}\right] \\
& -R_{2} \rho_{i} \rho_{k}-R_{3} \phi_{i} \phi_{k}
\end{aligned}
$$

In the above equations the parameters are defined as:

$$
\begin{aligned}
& m=\frac{\mu \gamma T_{0}}{D \rho_{l 0} L_{0}} ; \tilde{\epsilon}=\frac{h}{\xi} ; \alpha=\frac{\xi}{6 \sqrt{2} d_{0}} ; R_{1}=\frac{\mu \tau T_{0}}{6 \sqrt{2} h k \rho_{l 0} L_{0}} ; R_{2}=R_{3}=\frac{6 \sqrt{2} \gamma h}{\xi^{2} \rho_{l 0} v_{0}^{2}} ; \\
& R_{4}=\frac{\eta_{l}}{\tau \rho_{l 0} v_{0}^{2}} ; R_{5}=\frac{L_{0}}{v_{0}^{2}} ; R_{7}=\frac{\zeta_{l}}{\tau \rho_{l 0} v_{0}^{2}} ; R_{8}=\frac{\beta L_{0}}{C}
\end{aligned}
$$

where $\gamma$ is the surface tension, $h$ is the interface thickness, and $\mu$ is the kinetic undercooling coefficient that relates the interface undercooling to the interface 
velocity $v_{I}$ through $v_{I}=\mu\left(T_{0}-T\right)$. We indicate with $d_{0}=\left(\gamma C T_{0}\right) /\left(\rho_{0} L_{0}^{2}\right)$ the capillary length; $\eta_{l}, \zeta_{l}$ represent the first and second viscosity in the liquid. Anisotropy of the surface energy is accounted for through the function $\eta(\theta)=$ $(1+\omega \cos 4 \theta)$, where $\theta$ is defined as the angle between the normal to the interface and a fixed direction, the $x$ axis in our calculations, and $\omega$ specifies the intensity of the anisotropy. The numerical results presented in the following refer to the thermophysical properties of Nickel, with an interface thickness $h=30 \cdot 10^{-8} \mathrm{~cm}$. With a length scale $\xi=2 \cdot 10^{-4} \mathrm{~cm}$, the values of the model parameters are: $\alpha=265 ; m=0.1 ; \tilde{\epsilon}=1.5 \cdot 10^{-3} ; \omega=0.02 ; R_{1}=3.53 \cdot 10^{6} ; R_{2}=R_{3}=$ $1.07 \cdot 10^{-2} ; R_{4}=R_{7}=0.105 ; R_{5}=4.94 \cdot 10^{3}$. The numerical results are obtained neglecting thermal expansion effects $\left(R_{8}=0\right)$ and in absence of gravity.

\section{Numerical results}

Now we shall present numerical solutions of the model, in a two dimensional domain $\left(0 \leq x \leq x_{m}, 0 \leq y \leq y_{m}\right.$, with $\left.x_{m}=y_{m}=3.75\right)$. The boundary conditions are of Neumann type for the phase and temperature fields, and both permeable and impermeable for the mass flow.

\subsection{Growth of a free dendrite (permeable boundaries)}

In this set of solutions [3], the melt is initially at rest, at uniform temperature $u=-\Delta$, with $\Delta=0.7$; then a circular solid germ is nucleated at the center of the domain, with a supercritical radius $R_{0}=0.04$. In the first stage of the growth, the sudden contraction (or the expansion) of the liquid in front of the interface originates a pressure wave which propagates both into the solid and into the liquid. This effect is illustrated in Figures 1 and 2 where the pressure field is shown in the liquid at two different times; the crystal is represented as a black spot at the center of the graph. Here the density ratio is $S=1.05$, and we can clearly observe the large pressure drop in front of the dendrite tips. The numerical results are reported in non-dimensional units. The pressure front reaches the domain's boundaries, where it is reflected and redirected towards the crystal (Fig. 2).

In this stage we are far from a steady mechanical regime, nevertheless the shape of the crystal is already well defined, with steady values for both the tip velocity and the tip radius. However, the Peclet number $P=R_{\text {tip }} v_{\text {tip }} / 2 D$, which, according to the diffusive description of the process [4] should be only dependent on $\Delta$, is affected by the advection flow driven by the density change. We observed that the Peclet number is almost constant for $0.80 \leq S \leq 0.95$, then decreases with increasing $S$ between $0.95 \leq S \leq 1.20$.

\subsection{Growth in a closed system (constrained growth)}

Here we present numerical solutions [5] for the growth of a dendritic crystal with impermeable boundary conditions, assuming $S=0.85$, i.e. the liquid expands upon solidification. Then, the growth is contrasted by an increasing 


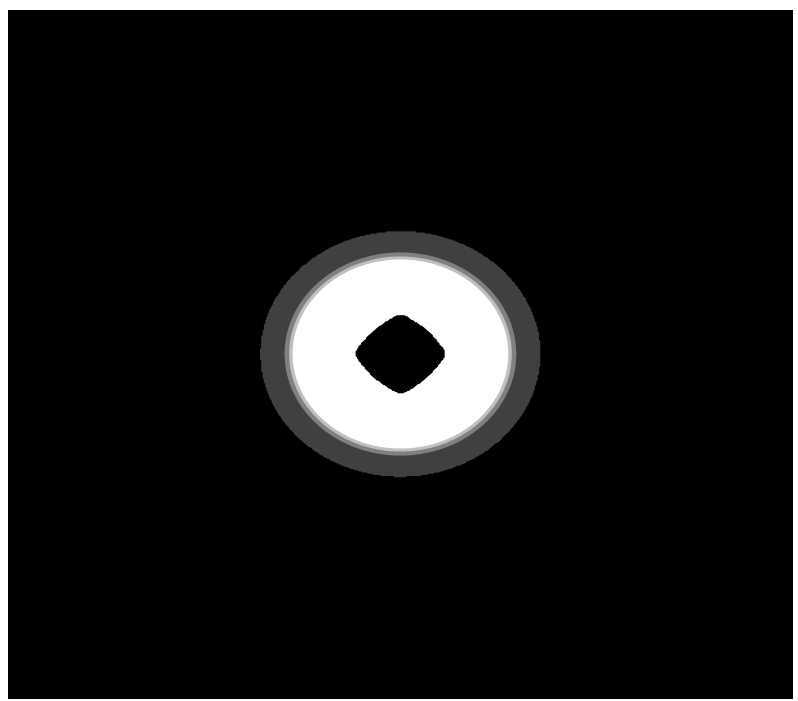

Figure 1: The pressure wave originated at the solid-liquid interface at time $0.81 \cdot 10^{-2}$. In order of increasing darkness the five zones represent pressure values $-25 \leq p \leq-20,-20 \leq p \leq-15,-15 \leq p \leq-5$, $-5 \leq p \leq-0.1,-0.1 \leq p \leq 0$.

pressure, which results in a continuous decrease of the coexistence temperature. In Figures 3 and 4 we represent, versus time, the tip velocity and the tip radius of the dendrite, for both free and constrained growth. We observe, for an open system, the well known steady regime. On the contrary, in a closed system the growth is characterized by a continuous decrease of the effective supercooling, and we observe that the tip velocity decreases with time and the tip radius increases.

An interesting question is whether in a closed system the growth follows a quasi-steady dynamics, adapting instantaneously to the variation of the effective supercooling. We observe that the Clapeyron's equation, in non-dimensional form, may be written as

$$
u_{\pi}=\frac{\tilde{\epsilon}}{\alpha} \frac{S-1}{S} \frac{1}{R_{3}}\left(p-p_{0}\right)
$$

related to the pressure shift $\left(p-p_{0}\right)$. The latter, averaged over the entire system, may be estimated as

$$
<p-p_{0}>=\frac{R_{1} R_{3}}{m}\left[\frac{1}{1+x_{s}(S-1)}-1\right]
$$

where $x_{s}$ is the actual solid fraction. The effective supercooling which drives the solidification, is $\Delta^{*}=\Delta+u_{\pi}$. We observed that along the growth, the Peclet number is well approximated by the values obtained for steady growth in an open system, with a supercooling $\Delta$ corresponding to the instantaneous values of $\Delta^{*}$. 


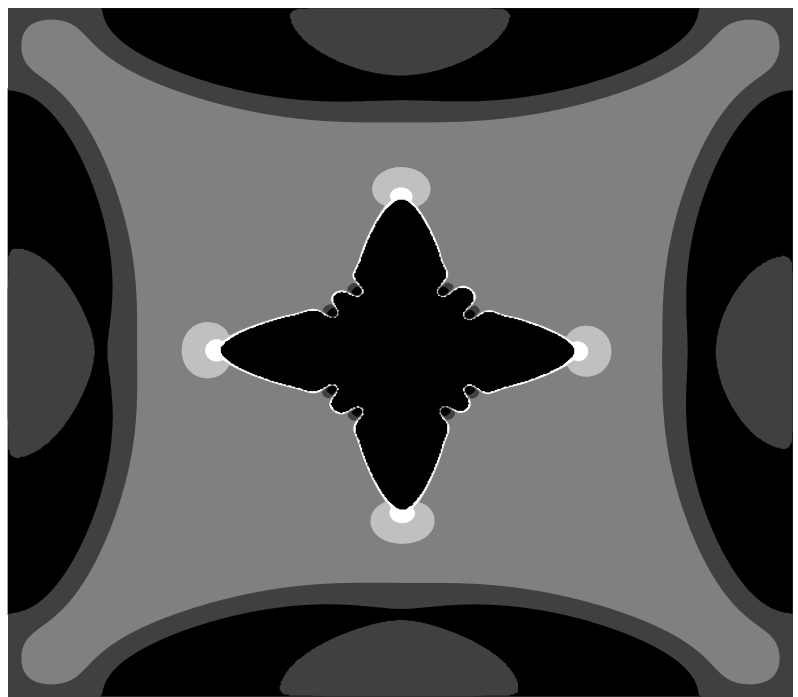

Figure 2: The pressure wave originated at the solid-liquid interface at time $4.05 \cdot 10^{-2}$. In order of increasing darkness the five zones represent pressure values $-25 \leq p \leq-20,-20 \leq p \leq-15,-15 \leq p \leq-5$, $-5 \leq p \leq-0.1,-0.1 \leq p \leq 0$.

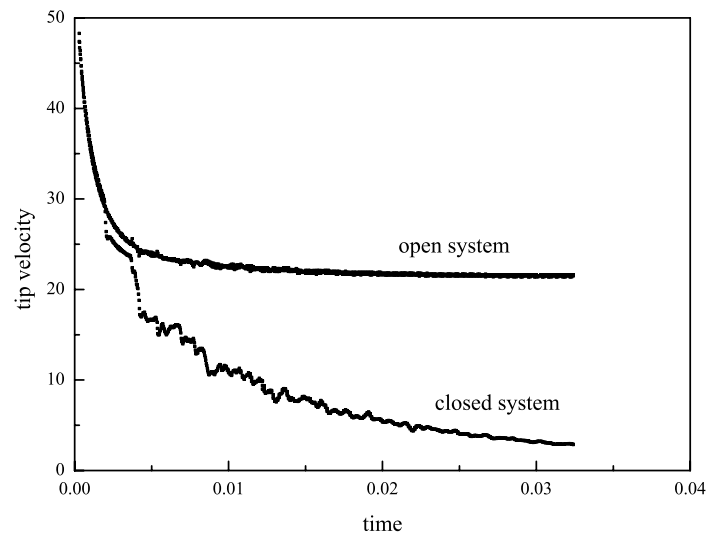

Figure 3: Dendritic growth. Tip velocity versus time in an open and a closed system.

\section{Conclusions}

The numerical solutions presented in this paper show to what extent the growth of a free dendrite deviates from the pure diffusive description of the process, when WIT Transactions on The Built Environment, Vol 92, (C) 2007 WIT Press

www.witpress.com, ISSN 1743-3509 (on-line) 


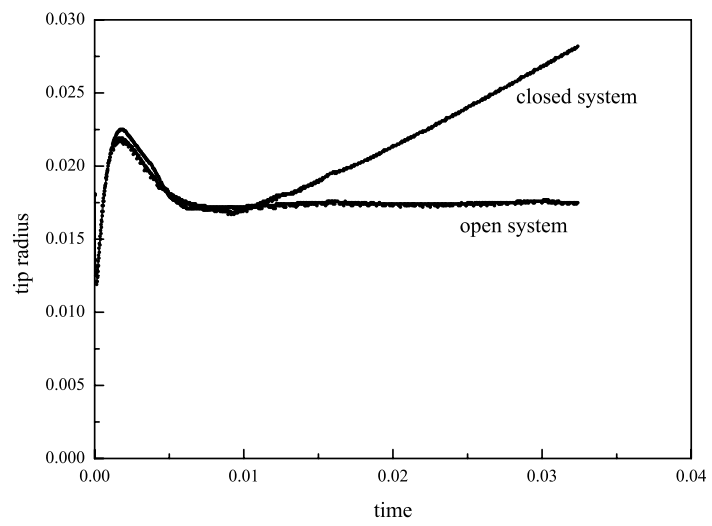

Figure 4: Dendritic growth. Tip radius versus time in an open and a closed system.

the advection flow effects are taken into account. Both the tip velocity and the tip radius, as well as the Peclet number, depend on the density ratio $S$.

We observed also that volumetric effects can influence constrained crystal growth in a significant manner. The melting temperature shift driven by the pressure change along the growth reduces the thermodynamic force available for solidification. In dendritic solidification the melting temperature shift destroys the steady regime, and the tip radius and velocity change with time. However, after the first fast transient, the Peclet number seems to be well approximated by the values obtained in steady conditions, with a supercooling corresponding to the instantaneous actual supercooling. This indicates that a quasi-steady approximation is a satisfactory approach to interpret the growth dynamics.

\section{References}

[1] G. Caginalp, P. Fife, Phase-field methods for interfacial boundaries, Phys. Rev. B, 33, (1986), pp. 7792-7794.

[2] M. Conti, Density effects and fluid flow in phase-field models, in H. Emmerich, B. Nestler, M. Schreckenberg, eds., Interface and Transport Dynamics, Springer, Berlin, (2003), pp. 121-135.

[3] M. Conti, Advection flow effects in the growth of a free dendrite, Phys.Rev. E, 69, (2004), pp. 022601-022604.

[4] W. Kurz and D. J. Fisher, Fundamentals of solidification (Trans Tech Publications, Aedermannsdorf, 1992).

[5] M. Conti, Pressure effects for crystal growth in a closed system, Phys.Rev. E, 70, (2004), pp. 1-6. 